\title{
Giant switchable Rashba effect in oxide heterostructures
}

\author{
Zhicheng Zhong ${ }^{1}$, Liang $\mathrm{Si}^{1}$, Qinfang Zhang ${ }^{2}$, Wei-Guo Yin ${ }^{3}$, Seiji Yunoki ${ }^{4}$ and Karsten Held ${ }^{1}$ \\ ${ }^{1}$ Institute of Solid State Physics, Vienna University of Technology, A-1040 Vienna, Austria \\ ${ }^{2}$ Key Laboratory for Advanced Technology in Environmental Protection \\ of Jiangsu Province, Yancheng Institute of technology, China \\ ${ }^{3}$ Condensed Matter Physics and Materials Science Department, \\ Brookhaven National Laboratory, Upton, New York 11973, USA \\ ${ }^{4}$ Computational Condensed Matter Physics Laboratory, RIKEN, Wako, Saitama 351-I0198, Japan
}

\begin{abstract}
One of the most fundamental phenomena and a reminder of the electron's relativistic nature is the Rashba spin splitting for broken inversion symmetry. Usually this splitting is a tiny relativistic correction, hardly discernible in experiment. Interfacing a ferroelectric, $\mathrm{BaTiO}_{3}$, and a heavy $5 d$ metal with a large spin-orbit coupling, $\mathrm{Ba}(\mathrm{Os}, \mathrm{Ir}) \mathrm{O}_{3}$, we show that giant Rashba spin splittings are indeed possible and even fully controllable by an external electric field. Based on density functional theory and a microscopic tight binding understanding, we conclude that the electric field is amplified and stored as a ferroelectric Ti-O distortion which, through the network of oxygen octahedra, also induces a large Os-O distortion. The $\mathrm{BaTiO}_{3} / \mathrm{BaOsO}_{3}$ heterostructure is hence the ideal test station for studying the fundamentals of the Rashba effect. It allows intriguing application such as the Datta-Das transistor to operate at room temperature.
\end{abstract}

PACS numbers: 73.20.-r, 73.21.-b, 79.60.Jv

An electric field control of the spin degree of freedom is the key to spintronics and magnetoelectrics [1]. In the prototypical spintronic device, the Datta-Das spin transistor [2], an electric field tunes the Rashba spin splitting and with that the spin precession frequency. The precession in turn controls the spin polarized current between two ferromagnetic leads. Microscopically, the Rashba spin splitting originates from the spin orbit coupling (SOC) in a two dimensional electron gas (2DEG) with broken inversion symmetry perpendicular to the 2DEG plane [3, 4]. It has been observed for metal surfaces $[5,6]$, semiconductor and oxide heterostructures [7-12], and even in polar bulk materials [13]. The Rashba effect splits parabolic bands into two subbands with opposite spin and energy-momentum dispersions $E^{ \pm}(\mathbf{k})=\left(\hbar^{2} \mathbf{k}^{2} / 2 m^{*}\right) \pm \alpha_{R}|\mathbf{k}|$. Here, $m^{*}$ is the effective mass, $\mathbf{k}$ the wave vector in the $2 \mathrm{DEG}$ plane, and $\alpha_{R}$ the Rashba coefficient which depends on the strength of SOC and inversion asymmetry. An electric field modulates this inversion asymmetry and consequently the Rashba spin splittings. The electric-field induced change of $\alpha_{R}$ is however weak: up to $10^{-2} \mathrm{eVA}$ in semiconductor [7] or $3 d$ transition metal oxide heterostructures [8-10]. A large, electric-field tunable Rashba effects are also much sought-after in the research area of topological insulators [14-16].

Giant Rashba effects with $\alpha_{R}$ of the order of $1 \mathrm{eVA}$ have been reported for metal surfaces [6], Bi adlayers [17] and bulk polar materials BiTeI $[13,18]$. The large $\alpha_{R}$ here relies on the surface or interface structural asymmetry, which is hardly changes in an external electric field. To enhance the tunability by an electric field, Di Sante et al. [19] hence suggested a ferroelectric semiconductor GeTe. For GeTe, an external electric field switches between paraelectric and ferroelectric phase, breaking in- version symmetry and tuning on the Rashba spin splitting. At first glance, this perfectly realizes an electric field control of a giant Rashba effect. However, there is an intrinsic difficulty: A single material cannot be both, a conductor with large Rashba spin splitting and a ferroelectric which necessarily is insulating.

In this letter, we propose to realize a giant switchable Rashba effect by heterostructures sandwiching a thin metallic film of heavy elements in-between ferroelectric insulators, see Fig. 1. The thin film provides a 2DEG with strong SOC, while the inversion asymmetry is induced by the structural distortion of the ferroelectrics and tunable by an electric field. As a prime example, we study a heterostructure of transition metals oxides, $\mathrm{BaOsO}_{3} / \mathrm{BaTiO}_{3} . \quad \mathrm{BaTiO}_{3}$ is a well-established ferroelectric [20]. It has a simple high-temperature perovskite structure, with $\mathrm{Ba}$ atoms at the edges, a Ti atom at the center and the $\mathrm{O}$ atoms at the faces of a cube. Such a structure has an inversion symmetry center at the $\mathrm{Ti}$ site. At room temperature, inversion symmetry is broken since a ferroelectric structural distortion occurs with a sizable Ti-O displacement $z_{\mathrm{Ti}-\mathrm{O}}$ along one of the the cubic axes. $\mathrm{BaOsO}_{3}$ has been recently synthesized; it is a metallic perovskite with four Os $5 d$ electrons and no sign of magnetism [21]. It has a perfect lattice match with $\mathrm{BaTiO}_{3}$. Both materials have the same cation, $\mathrm{Ba}$, which will substantially reduce disorder during epitaxial growth. For $\mathrm{BaOsO}_{3} / \mathrm{BaTiO}_{3}$ we find an electric-field tunable Rashba spin splitting which is at least one magnitude larger than the current experimental record [7-10]. The mechanism behind is nontrivial and summarized in Fig. 1. Substituting $\mathrm{BaOsO}_{3}$ by $\mathrm{BaIrO}_{3}$ and $\mathrm{BaRuO}_{3}$ yields a similar effect; the heterostructure can also be further engineered by varying its thickness and strain.

Method. We mainly focus on a $\mathrm{BaOsO}_{3} / \mathrm{BaTiO}_{3}$ het- 
(3) orbital deformation

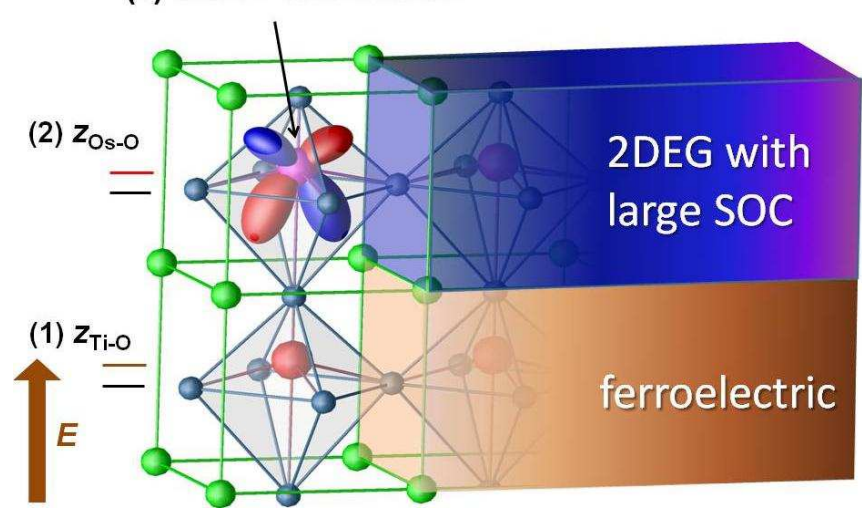

FIG. 1: Schematics of the mechanism behind the giant switchable Rashba effect: (1) An external electric field is amplified and stored as a ferroelectric distortion, in particular Ti-O displacements. (2) These displacements entail, via the oxygen octahedron network of the perovskite heterostructure, Os-O displacements. (3) As a consequence of the Os-O displacements the Os orbitals deform, which reflects the broken inversion symmetry. This orbital deformation and the strong SOC of Os finally lead to a giant Rashba spin splittings that is controllable by an electric-field.

erostructure which has two structural phases: a paraelectric perovskite phase and a distorted ferroelectric phase, see Fig. 2(c) and (d), respectively. We use densityfunctional theory (DFT) with generalized gradient approximation (GGA) potential [22] in the Vienna Ab initio Simulation Package (VASP) [23, 24] and fully relax all the atomic positions, only fixing the paraelectric or ferroelectric symmetries. At zero temperature, the ferroelectric distorted perovskite has the lower GGA energy, but -as in the bulk- the undistorted paraelectric phase will prevail at elevated temperatures.

Based on the fully relaxed atomic structures, electronic band structures are calculated with modified BeckeJohnson (mBJ) [25] exchange potential as implemented in the Wien $2 \mathrm{k}$ code [26], which improves the calculated bandgap of $\mathrm{BaTiO}_{3}$. The SOC is included as a perturbation using the scalar-relativistic eigenfunctions of the valence states. Employing wien2wannier [27], we project the Wien2k bandstructure onto maximally localized[28] Wannier orbitals, from which the orbital deformation is analyzed and a realistic tight binding model is derived [29]. We also vary the thickness of the thin films, replace the $\mathrm{BaOsO}_{3}$ by $\mathrm{BaRuO}_{3}$ or $\mathrm{BaIrO}_{3}$, and simulate the strain effect by fixing the in plane lattice constant to the value of a $\mathrm{SrTiO}_{3}$ substrate. Correlation effects from local Coulomb interaction are studied in the Supplementary Material.

Paraelectric phase. We mainly focus on a $\mathrm{BaOsO}_{3} / \mathrm{BaTiO}_{3}$ heterostructure, $\left(\mathrm{BaOsO}_{3}\right)_{1} /\left(\mathrm{BaTiO}_{3}\right)_{4}$, which consists of one $\mathrm{BaOsO}_{3}$ layer alternating with four $\mathrm{BaTiO}_{3}$ layers. Bulk $\mathrm{BaTiO}_{3}$ is a $d^{0}$ insulator: the empty Ti $3 d$ states lie about $3 \mathrm{eV}$ above occupied $\mathrm{O} 2 p$ states. Bulk $\mathrm{BaOsO}_{3}$ is a $d^{4}$ metal: four electrons in the Os $5 d$ orbitals of $t_{2 g}$ character are $0.8 \mathrm{eV}$ above the filled $\mathrm{O} 2 p$ states. Since $\mathrm{BaOsO}_{3}$ and $\mathrm{BaTiO}_{3}$ share oxygen atoms at the interface, the $\mathrm{O} 2 p$ states align; and the Os $5 d$ states will stay in the energy gap of $\mathrm{BaTiO}_{3}$. Hence, the density functional theory (DFT) calculated band structures of $\mathrm{BaOsO}_{3} / \mathrm{BaTiO}_{3}$ in Fig. 2 shows three Os $t_{2 g}(x y, y z, x z)$ bands near the Fermi level; they are dispersionless along the $z$ direction (not shown). That is, the low energy electronic degrees of freedom are confined by the insulating $\mathrm{BaTiO}_{3}$ layers to the $\mathrm{OsO}_{2}$ plane, forming a 2DEG. Already in the high temperature paraelectric phase, this heterostructure confinement reduces the initial cubic symmetry of the Os $t_{2 g}$ orbitals in the bulk perovskite: At $\Gamma$ the $x y$ band in Fig. 2 (a) is $1.2 \mathrm{eV}$ lower in energy than the degenerate $y z / x z$ bands (Fig. 2(a)). Including the SOC, the $y z / x z$ doublet splits into two subbands with mixed $y z / x z$ orbital character, see Fig. 2(b). For the paraelectric phase of Fig. 2(a-c), the spin degeneracy is however still preserved.

To better understand the DFT results, we construct an interface hopping Hamiltonian $H_{0}^{i}$ [30] for the 2DEG confined Os $t_{2 g}$ electrons by a Wannier projection. The energy-momentum dispersion for the $x y$ orbital is $\epsilon(\mathbf{k})^{x y}=-2 t_{1} \cos k_{x}-2 t_{1} \cos k_{y}-4 t_{3} \cos k_{x} \cos k_{y}$, while that of the $y z$ is $\epsilon(\mathbf{k})^{y z}=-2 t_{2} \cos k_{x}-2 t_{1} \cos k_{y}\left(\epsilon(\mathbf{k})^{x z}\right.$ is symmetrically related by $x \leftrightarrow y)$. The largest hopping $t_{1}=0.392 \mathrm{eV}$ is along the direction(s) of the orbital lobes, $t_{2}=0.033 \mathrm{eV}$ and $t_{3}=0.094 \mathrm{eV}$ indicate a much smaller hopping perpendicular to the lobes and along $(1,1,0)$, respectively. The $x y-y z / x z$ orbital splitting at $\Gamma$ is $2 t_{1}-2 t_{2}+4 t_{3}=1.1 \mathrm{eV}$ and arises from the anisotropy of the orbitals. Overall, the three parameter tight binding model in Fig. 3(a) (dashed line) is consistent with DFT in Fig. 2(a). Next, we include the atomic SOC Hamiltonian $H_{\xi}=\xi \vec{l} \cdot \vec{s}$, expressed in the $t_{2 g}$ basis with $\xi=0.44 \mathrm{eV}$ for Os. It lifts the $y z / x z$ degeneracy and yields good agreement with DFT, cf. Fig. 3(a) (solid line) and Fig. 2(b).

Ferroelectric phase. In sharp contrast to the paraelectric case, ferroelectrically distorted $\mathrm{BaOsO}_{3} / \mathrm{BaTiO}_{3}$ exhibits evident spin splittings in the presence of SOC, see Fig. 2(f). Note, in the absence of SOC the DFT band structure in Fig. 2(e) is still very similar to the paraelectric case in Fig. 2(a). The spin splitting $E_{\mathrm{R}}^{x y}$ of the $x y$ band is small around $\Gamma$, but strongly enhanced up to $50 \mathrm{meV}$ around the $x y / y z$ crossing region as shown in Fig. 2(g), cf. Table I, . The other two subbands of $y z / x z$ character also show a strong splitting around $\Gamma$. In this respect, the upper $y z / x z$ subband exhibits a standard Rashba behavior, see the magnification in Fig. 2(h), where the momentum offset $k_{0}=$ $0.043 \AA^{-1}$, the Rashba energy $E_{\mathrm{R}}=4 \mathrm{meV}$, and hence $\alpha_{\mathrm{R}}=2 E_{\mathrm{R}} / k_{0}=0.186 \mathrm{eVA}$. The behavior of the lower 

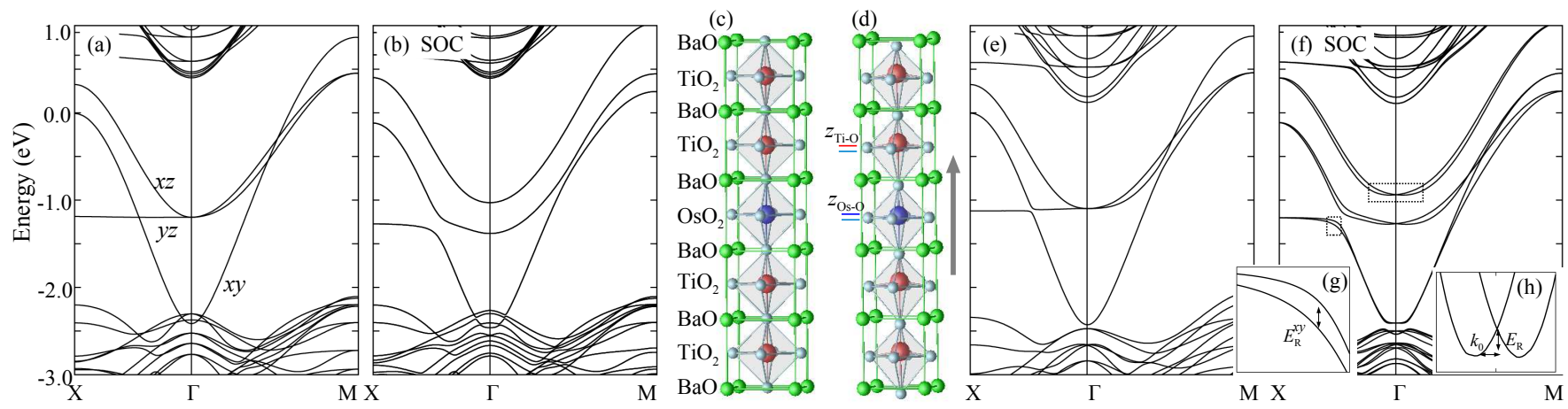

FIG. 2: DFT calculated band structures of $\left(\mathrm{BaOsO}_{3}\right)_{1} /\left(\mathrm{BaTiO}_{3}\right)_{4}$ multilayers for the paraelectric (c) and ferroelectric structure (d). The paraelectric heterostructure breaks cubic but not inversion symmetry so that the Os $x y$ orbital splits off from two degenerate $y z / x z$ orbitals without SOC (a); including SOC further lifts the $y z / x z$ degeneracy (b). The ferroelectric state further breaks inversion symmetry, which essentially does not change the bandstructure in the absence of SOC (e) but leads to substantial spin splittings in the presence of SOC (f). The spin splitting $E_{\mathrm{R}}^{x y}$ at the $x y-y z$ crossing region is magnified in (g), while the standard Rashba spin splitting $E_{\mathrm{R}}$ with momentum offset $k_{0}$ around $\Gamma$ of the upper $y z / x z$ mixed orbital is magnified in $(\mathrm{h})$
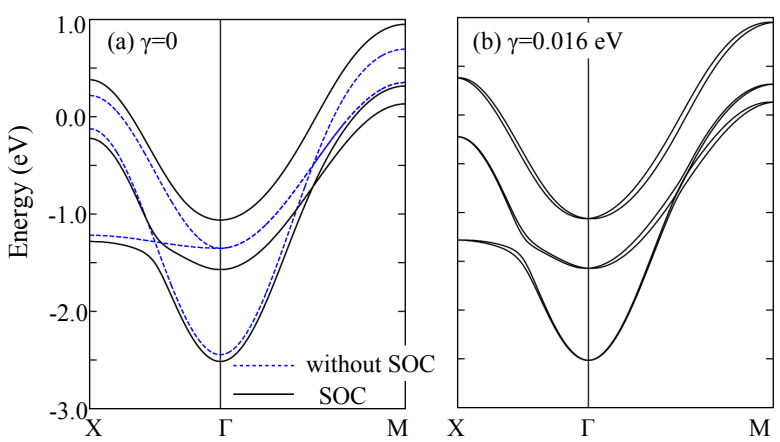

FIG. 3: Tight binding bandstructure of the three Os $t_{2 g}$ orbitals for (a) the paraelectric case with and without SOC and (b) the ferroelectric case with SOC and asymmetry parameter $\gamma=0.016 \mathrm{eV}$ taken from the Wannier projection.

$y z / x z$ subband is more complex (distorted) due to the proximity of the crossing region.

These spin splittings arise from ferroelectric distortions which break the inversion symmetry. Bulk $\mathrm{BaOsO}_{3}$ has no such ferroelectric distortions and is inversion symmetric with $\mathrm{Os}$ and $\mathrm{O}$ atom in the same plane. Also for the paraelectric heterostructure, the $\mathrm{OsO}_{2}$ layer is still an inversion plane. The ferroelectric heterostructure breaks inversion symmetry. As listed in Table I, the averaged ferroelectric Ti-O displacement in $\mathrm{BaTiO}_{3}$ layers is around $0.14 \AA$, which efficiently induces a sizable Os-O displacement of $0.05 \AA$ via the oxygen octahedron network of the perovskite structure. This structural distortion modifies the crystal environment of Os $t_{2 g}$ orbitals and deforms the orbital lobes, see Fig. 1.

In order to quantify the orbital deformation, we project the DFT results above onto maximally localized Wannier orbitals and directly extract the key parameter: a directional, spin-independent inter-orbital hopping $\gamma=$ $\langle x y|H| y z(R)\rangle$, were $R$ is the nearest neighbor in $x$ direc- tion. In $\mathbf{k}$-space one gets as a matrix for the $y z, x z, x y$ orbitals (independent of spin) [29]

$$
H_{\gamma}=\gamma\left(\begin{array}{ccc}
0 & 0 & 2 i \sin k_{x} \\
0 & 0 & 2 i \sin k_{y} \\
-2 i \sin k_{x} & -2 i \sin k_{y} & 0
\end{array}\right) .
$$

We obtain $\gamma=0.016 \mathrm{eV}$ for the ferroelectric heterostructure, while for the paraelectric case $\gamma=0$ due to inversion symmetry. Let us emphasize that $\gamma$ is the relevant measure for the orbital deformation and inversion symmetry breaking. In combination with the strong SOC of OS $5 d$ electrons, $H_{\gamma}$ results in the present giant Rashba spin splitting.

The Hamiltonian $H_{0}^{i}+H_{\xi}+H_{\gamma}$ already well describes the spin splittings of the $x y$ orbital in good agreement with DFT results, but underestimates the spin splittings of the $y z / x z$ subbands near $\Gamma$. The reason for this is that the atomic SOC is not an accurate description anymore because the SOC of Os is too strong and the deviation from the inversion symmetry too large. Hence, we need to go beyond the purely atomic SOC and include an inversion asymmetry correction to the SOC matrix so that $H_{\xi}$ in the $t_{2 g}$ basis $(y z|\uparrow\rangle, y z|\downarrow\rangle, x z|\uparrow\rangle, x z|\downarrow\rangle, x y|\uparrow\rangle$, $x y|\downarrow\rangle)$ reads

$$
\left(\begin{array}{cccccc}
0 & 2 \gamma^{\prime} \sin k_{y} & i \frac{\xi}{2} & 0 & 0 & -\frac{\xi}{2} \\
2 \gamma^{\prime} \sin k_{y} & 0 & 0 & -i \frac{\xi}{2} & \frac{\xi}{2} & 0 \\
-i \frac{\xi}{2} & 0 & 0 & -2 \gamma^{\prime} \sin k_{x} & 0 & i \frac{\xi}{2} \\
0 & i \frac{\xi}{2} & -2 \gamma^{\prime} \sin k_{x} & 0 & i \frac{\xi}{2} & 0 \\
0 & \frac{\xi}{2} & 0 & -i \frac{\xi}{2} & 0 & 0 \\
-\frac{\xi}{2} & 0 & -i \frac{\xi}{2} & 0 & 0 & 0
\end{array}\right)
$$

For the bandstructure of Fig. 3(b) we have taken $\gamma^{\prime}=$ $0.022 \mathrm{eV}$ from the Wannier projection, yielding good agreement with the DFT results Fig. 2(f). 
TABLE I: Polar distortions and Rashba spin splittings of $\left(\mathrm{BaOsO}_{3}\right)_{n} /\left(\mathrm{BaTiO}_{3}\right)_{m}$ multilayers, alternating $n$ layers of $\mathrm{BaOsO}{ }_{3}$ and $m$ layers of $\mathrm{BaTiO}_{3}$. Second and third column: averaged displacement of Os-O and Ti-O along $z$ as calculated by DFT. Forth column: strength of the interface asymmetry term $\gamma$ obtained from the Wannier projection. Fifth to seventh column: DFT calculated parameters characterizing Rashba effects: momentum offset $k_{0}$, Rashba energy $E_{\mathrm{R}}$, and Rashba coefficient $\alpha_{\mathrm{R}}$ of the upper $y z / x z$ subband around $\Gamma$ as defined in Fig. 2(h). Eighth column: spin splitting $E_{\mathrm{R}}^{x y}$ around the crossing region of $x y$ and $y z$ orbitals as in Fig. 1(g). To demonstrate the effects of heterostructure engineering, we also list the results of $\mathrm{BaOsO}_{3} / \mathrm{BaTiO}_{3}$ strained by a $\mathrm{SrTiO}_{3}$ substrate, as well as $\mathrm{BaRuO}_{3} / \mathrm{BaTiO}_{3}$ and $\mathrm{BaIrO}_{3} / \mathrm{BaTiO}_{3}$. Band structures are given in Supplementary Material.

\begin{tabular}{llllllll}
\hline \hline$n: m$ & Os-O $(\AA)$ & Ti-O $(\AA)$ & $\gamma(\mathrm{eV})$ & $k_{0}\left(\AA^{-1}\right)$ & $E_{\mathrm{R}}(\mathrm{eV})$ & $\alpha_{\mathrm{R}}(\mathrm{eV} \AA)$ & $E_{\mathrm{R}}^{x y}(\mathrm{eV})$ \\
\hline $1: 3 \mathrm{BaOsO}_{3} / \mathrm{BaTiO}_{3}$ & 0.038 & 0.065 & 0.011 & 0.025 & 0.001 & 0.08 & 0.031 \\
$1: 4$ & 0.049 & 0.103 & 0.016 & 0.043 & 0.004 & 0.186 & 0.053 \\
$2: 3$ & $<0.010$ & $<0.010$ & 0.003 & 0 & 0 & - & 0.004 \\
$2: 4$ & 0.024 & 0.071 & 0.010 & 0.035 & 0.002 & 0.114 & 0.018 \\
$1: 3$ strained & 0.095 & 0.223 & 0.030 & 0.105 & 0.021 & 0.396 & 0.102 \\
$1: 4$ strained & 0.099 & 0.229 & 0.031 & 0.115 & 0.024 & 0.417 & 0.106 \\
\hline $1: 4 \mathrm{BaIrO}_{3} / \mathrm{BaTiO}_{3}$ & 0.115 & 0.119 & 0.021 & 0.145 & 0.053 & 0.731 & 0.051 \\
$1: 4 \mathrm{BaRuO}_{3} / \mathrm{BaTiO}_{3}$ & 0.082 & 0.119 & 0.015 & 0.128 & 0.016 & 0.250 & 0.007 \\
\hline \hline
\end{tabular}

Electric-field tunability. The Rashba spin splittings can be tuned by an electric field because of the ferroelectricity of $\mathrm{BaTiO}_{3}$. It is a nature of ferroelectrics that an external electric field can tune and even reverse the polarization and structural distortion [17, 20, 31-33], including that of the $\mathrm{BaOsO}_{3}$ layer. Because of the $\mathrm{BaOsO}_{3}$ layer, the ferroelectricity of the heterostructure is not as strong as in bulk $\mathrm{BaTiO}_{3}$. Hence a smaller electric field change than in the bulk $\left(\mathrm{E}=10^{3} \mathrm{~V} / \mathrm{cm}[33]\right)$ is be needed for going through the ferroelectric hysteresis loop. Such an electric field can tune the Rashba coefficient listed in Table I, which are at least one order of magnitude more larger than in semiconductors [7] or $3 d$ oxide heterostructures [8-10]. For applications it is of particular importance that $E_{R}$ now clearly exceeds $0.025 \mathrm{eV}$, the thermal energy at room temperature. Furthermore, we propose to take advantage of the ferroelectric hysteresis and the remnant ferroelectric polarization, using our heterostructure as a spintronics memory device.

Heterostructure engineering. Besides applying an electric field, heterostructure engineering such as varying its thickness, strain, and the material combination is a powerful way to tune the Rashba spin splittings. First, we vary the thickness of $\mathrm{BaOsO}_{3}$ and $\mathrm{BaTiO}_{3}$. It is well known experimentally and theoretically $[20,34]$ that below a few nanometer thickness of the $\mathrm{BaTiO}_{3}$ film, the Ti$\mathrm{O}$ displacement is reduced and eventually ferroelectricity vanishes. As shown in Table I, when decreasing the thickness of $\mathrm{BaTiO}_{3}$ and increasing that of $\mathrm{BaOsO}_{3}$, the Rashba spin splittings is indeed substantially reduced. Second, ferroelectricity of $\mathrm{BaTiO}_{3}$ is sensitive to strain [35]. Taking $\mathrm{SrTiO}_{3}$ as a substrate provides a $2.5 \%$ inplane compressive strain. This enhances the ferroelectric distortion as well as Rashba spin splitting, $\alpha_{\mathrm{R}}=0.417 \mathrm{eV \AA}$ in Table I. Given the giant Rashba splitting, photoemission and transport measurements should be able to validate this effect by comparing thin films with different thickness and substrate. Third, we replace Os by other $5 d$ elements for shifting the Fermi energy; and also since some Os oxides are toxic. Indeed, similar physics can be found in $\mathrm{BaIrO}_{3} / \mathrm{BaTiO}_{3}$ with $\alpha_{\mathrm{R}}=0.731 \mathrm{eV} \AA$, see Table I, and in $\mathrm{BaRuO}_{3} / \mathrm{BaTiO}_{3}$. The latter also offers a platform to study the interplay of Rashba physics and electronic correlations; for instance, novel phenomena is expected in mixed $3 d-5 d$ transition-metal materials [36]. Note a strong temperature dependence of the $\alpha_{R}$ in $\mathrm{SrIrO}_{3}$ was recently reported [37].

Conclusion. We propose to combine a heavy $5 d$ compound such as $\mathrm{BaOsO}_{3}$ and a ferroelectric such as $\mathrm{BaTiO}_{3}$ in a heterostructure for realizing giant Rashba spin splittings that are switchable by an electric field. The physics behind is that ferroelectric $\mathrm{BaTiO}_{3}$ amplifies the electric field through a distortion (polarization) of its $\mathrm{TiO}_{6}$ octahedra. This distortion very efficiently propagates to the $\mathrm{OsO}_{6}$ octahedron via the shared oxygens, breaking inversion symmetry and deforming the Os orbital lobes. Together with the strong SOC it leads to a giant tunable Rashba effect with, e.g., $\alpha_{\mathrm{R}}=0.731 \mathrm{eV} \AA$ for $\mathrm{BaIrO}_{3} / \mathrm{BaTiO}_{3}$. This is at least one magnitude larger than state-of-the-art. Moreover, the ferroelectric hysteresis and remnant polarization leads to a memory effect which can be exploited for spintronics memory devices.

Acknowledgments. ZZ acknowledges financial support by the Austrian Science Fund through the SFB ViCoM F4103-N13, QFZ by NSFC (11204265), the NSF of Jiangsu Province (BK2012248), KH by the European Research Council under the European Union's Seventh Framework Program (FP/2007-2013)/ERC through grant agreement n. 306447, and WY by the U.S. Department of Energy under Contract No. DE-AC0298CH10886. Calculations have been done on the Vienna Scientific Cluster (VSC). 
[1] I. Žutić, J. Fabian, and S. Das Sarma, Rev. Mod. Phys. 76, 323 (2004).

[2] S. Datta and B. Das, Appl. Phys. Lett. 56, 665 (1990).

[3] E. I. Rashba, Sov. Phys. Solid State 2, 1109 (1960).

[4] R. Winkler, Spin Orbit Coupling Effects in TwoDimensional Electron and Hole Systems (Springer, 2003).

[5] S. LaShell, B. A. McDougall, and E. Jensen, Phys. Rev. Lett. 77, 3419 (1996).

[6] C. R. Ast, J. Henk, A. Ernst, L. Moreschini, M. C. Falub, D. Pacilé, P. Bruno, K. Kern, and M. Grioni, Phys. Rev. Lett. 98, 186807 (2007).

[7] J. Nitta, T. Akazaki, H. Takayanagi, and T. Enoki, Phys. Rev. Lett. 78, 1335 (1997).

[8] A. D. Caviglia, M. Gabay, S. Gariglio, N. Reyren, C. Cancellieri, and J.-M. Triscone, Phys. Rev. Lett. 104, 126803 (2010).

[9] M. Ben Shalom, M. Sachs, D. Rakhmilevitch, A. Palevski, and Y. Dagan, Phys. Rev. Lett. 104, 126802 (2010).

[10] H. Nakamura, T. Koga, and T. Kimura, Phys. Rev. Lett. 108, 206601 (2012).

[11] P. D. C. King, R. C. Hatch, M. Bianchi, R. Ovsyannikov, C. Lupulescu, G. Landolt, B. Slomski, J. H. Dil, D. Guan, J. L. Mi, et al., Phys. Rev. Lett. 107, 096802 (2011).

[12] P. D. C. King, R. H. He, T. Eknapakul, P. Buaphet, S.-K. Mo, Y. Kaneko, S. Harashima, Y. Hikita, M. S. Bahramy, C. Bell, et al., Phys. Rev. Lett. 108, 117602 (2012).

[13] K. Ishizaka, M. S. Bahramy, H. Murakawa, M. Sakano, T. Shimojima, T. Sonobe, K. Koizumi, S. Shin, H. Miyahara, A. Kimura, et al., Nat. Mat. 10, 521 (2011).

[14] M. Bahramy, B. Yang, R. Arita, and N. Nagaosa, Nat. Commun. 3, 679 (2012).

[15] S. Nakosai, Y. Tanaka, and N. Nagaosa, Phys. Rev. Lett. 108, 147003 (2012).

[16] T. Das and A. V. Balatsky, Nat. Commun. 4, 1972 (2013).

[17] H. Mirhosseini, I. V. Maznichenko, S. Abdelouahed, S. Ostanin, A. Ernst, I. Mertig, and J. Henk, Phys. Rev. B 81, 073406 (2010).

[18] J.-J. Zhou, W. Feng, Y. Zhang, and S. A. Y. andYugui Yao, Scientific Reports 4, 3841 (2014).

[19] D. Di Sante, P. Barone, R. Bertacco, and S. Picozzi, Ad- vanced Materials 25, 509 (2013), ISSN 1521-4095.

[20] M. Dawber, K. M. Rabe, and J. F. Scott, Rev. Mod. Phys. 77, 1083 (2005).

[21] Y. Shi, Y. Guo, Y. Shirako, W. Yi, X. Wang, A. A. Belik, Y. Matsushita, H. L. Feng, Y. Tsujimoto, M. Arai, et al., Journal of the American Chemical Society 135, 16507 (2013).

[22] J. P. Perdew, K. Burke, and M. Ernzerhof, Phys. Rev. Lett. 77, 3865 (1996).

[23] G. Kresse and J. Hafner, Phys. Rev. B 47, 558 (1993).

[24] G. Kresse and J. Furthmüller, Phys. Rev. B 54, 11169 (1996).

[25] F. Tran and P. Blaha, Phys. Rev. Lett. 102, 226401 (2009).

[26] P. Blaha, K. Schwarz, G. K. H. Madsen, D. Kvasnicka, and J. Luitz, WIEN2k, An Augmented Plane Wave + Local Orbitals Program for Calculating Crystal Properties (Karlheinz Schwarz, Techn. Universität Wien, Austria, 2001), ISBN 3-9501031-1-2.

[27] J. Kune, R. Arita, P. Wissgott, A. Toschi, H. Ikeda, and K. Held, Computer Physics Communications 181, 1888 (2010), ISSN 0010-4655.

[28] A. A. Mostofi, J. R. Yates, Y.-S. Lee, I. Souza, D. Vanderbilt, and N. Marzari, Computer Physics Communications 178, 685 (2008), ISSN 0010-4655.

[29] Z. Zhong, A. Tóth, and K. Held, Phys. Rev. B 87, 161102 (2013).

[30] Z. Zhong, Q. Zhang, and K. Held, Phys. Rev. B 88, 125401 (2013).

[31] S. Mathews, R. Ramesh, T. Venkatesan, and J. Benedetto, Science 276, 238 (1997).

[32] C.-G. Duan, S. S. Jaswal, and E. Y. Tsymbal, Phys. Rev. Lett. 97, 047201 (2006).

[33] R. Tazaki, D. Fu, M. Itoh, M. Daimon, and S. ya Koshihara, J. Phys.: Condens. Matter. 21, 215903 (2009).

[34] J. Junquera and P. Ghosez, Nature 422, 506 (2003).

[35] K. J. Choi, M. Biegalski, Y. L. Li, A. Sharan, J. Schubert, R. Uecker, P. Reiche, Y. B. Chen, X. Q. Pan, V. Gopalan, et al., Science 306, 1005 (2004).

[36] W.-G. Yin, X. Liu, A. M. Tsvelik, M. P. M. Dean, M. H. Upton, J. Kim, D. Casa, A. Said, T. Gog, T. F. Qi, et al., Phys. Rev. Lett. 111, 057202 (2013).

[37] L. Zhang, Y. B. Chen, J. Zhou, S.-T. Zhang, Z. bin Gu, S.-H. Yao, and Y.-F. Chen, arXiv 1309.7566 (2013). 